\title{
Spectral Properties of Quarks in the Quark-Gluon Plasma
}

\section{Frithjof Karsch}

Brookhaven National Laboratory, Bldg.510A, Upton, 11973, NY, USA

E-mail: karsch@quark.phy.bnl.gov

\section{Masakiyo Kitazawa*}

Department of Physics, Osaka University, Toyonaka, Osaka, 560-0043, Japan

E-mail: kitazawa@phys.sci.osaka-u.ac.jp

\begin{abstract}
We analyze the spectral properties of the quark propagator above the critical temperature for the deconfinement phase transition in quenched lattice QCD using clover improved Wilson fermions. The bare quark mass dependence of the quark spectral function is analyzed by varying the hopping parameter $\kappa$ in Landau gauge. We assume a two-pole structure for the quark spectral function, which is numerically found to work quite well for any value of $\kappa$. It is shown that in the chiral limit the quark spectral function has two collective modes that correspond to the normal and plasmino excitations, while it is dominated by a single-pole structure when the bare quark mass becomes large.
\end{abstract}

The XXV International Symposium on Lattice Field Theory

July 30-4 August 2007

Regensburg, Germany

\footnotetext{
*Speaker.
} 


\section{Introduction}

Hadronic matter undergoes a phase transition to a deconfined phase at nonzero temperature. The properties of the phase above the critical temperature, $T_{c}$, acquires much interest both from the experimental and theoretical point of view. In order to understand the structure of the matter in this region, it is desirable to identify the basic degrees of freedom of the system and their quasi-particle properties. In the present study, we analyze dynamical properties of quarks above $T_{c}$ in quenched lattice QCD in Landau gauge [1].

At asymptotically high temperatures one can calculate the quark propagator using perturbative techniques. It is known that the collective excitations of quarks in this limit develop a mass gap (thermal mass) that is proportional to $g T$ [2, 3]. Here $g$ and $T$ denote the gauge coupling and temperature, respectively. Moreover, in this limit the number of poles in the quark propagator is doubled. In addition to the normal modes, which reduce to poles in the free particle propagator, plasmino modes appear.

In order to understand the origin of the plasmino mode in the quark propagator in the high temperature limit, it is instructive to consider the quark propagator at intermediate temperature [4, 5]. In [4] the temperature dependence of the spectral function for fermions with scalar mass $m$ has been considered in QED and a Yukawa model. In these models the spectral function at zero temperature has two poles at energies $\omega= \pm m$, while in the high temperature limit, $T / m \rightarrow \infty$, it approaches the propagator having four poles. The one-loop calculation performed in [4] clearly showed that the two limiting forms of the spectral function are connected continuously; in addition to the normal quasi-particle peak a peak corresponding to the plasmino gradually appears in the spectral function and becomes larger with increasing temperature, [4]. In the present study, we analyze the quark propagator in quenched lattice QCD at two values of the temperature, $T=1.5 T_{c}$ and $3 T_{c}$, as a function of the bare quark mass. To simplify the present analysis, all our calculations have been performed for zero momentum.

\section{Quark Spectral Function}

The dynamical properties of quarks at zero momentum are encoded in the quark spectral function $\rho(\omega)$ which is related to the Euclidean correlation function

$$
S(\tau)=\frac{1}{V} \int d^{3} x d^{3} y\langle\psi(\tau, x) \bar{\psi}(0, y)\rangle
$$

through an integral equation

$$
S(\tau)=\int_{-\infty}^{\infty} d \omega \frac{e^{(1 / 2-\tau T) \omega / T}}{e^{\omega / 2 T}+e^{-\omega / 2 T}} \rho(\omega),
$$

with the quark field $\psi$, the spatial volume $V$, and the imaginary time $\tau$ which is restricted to $0 \leq \tau<1 / T$. The Dirac structure of $\rho(\omega)$ is decomposed as

$$
\begin{aligned}
\rho(\omega) & =\rho_{0}(\omega) \gamma^{0}+\rho_{\mathrm{s}}(\omega) \\
& =\rho_{+}(\omega) \Lambda_{+} \gamma^{0}+\rho_{-}(\omega) \Lambda_{-} \gamma^{0}
\end{aligned}
$$




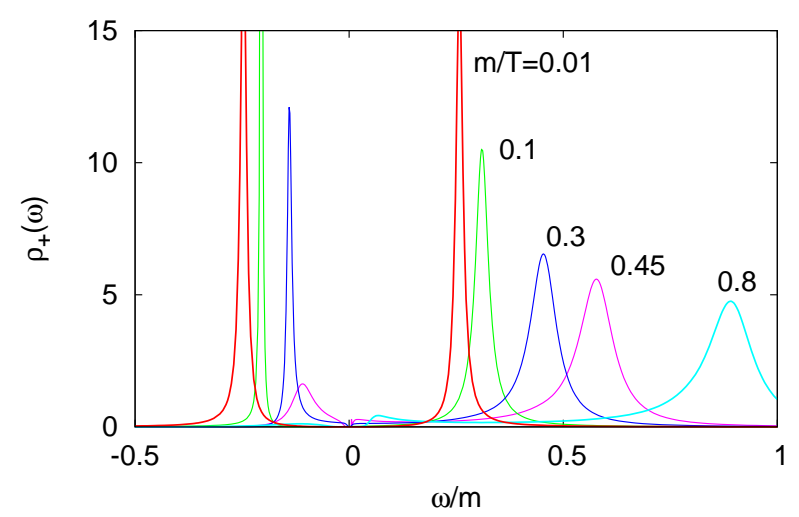

Figure 1: The spectral function $\rho_{+}(\omega)$ in the Yukawa model with massive fermion and massless boson for various values of $m / T$ [4].

\begin{tabular}{crrcccc}
\hline \hline$T / T_{c}$ & $N_{\tau}$ & $N_{\sigma}$ & $\beta$ & $c_{\mathrm{SW}}$ & $\kappa_{c}$ & $a[\mathrm{fm}]$ \\
\hline 3 & 16 & 64,48 & 7.457 & 1.3389 & 0.13390 & 0.015 \\
& 12 & 48 & 7.192 & 1.3550 & 0.13437 & 0.021 \\
1.5 & 16 & 64,48 & 6.872 & 1.4125 & 0.13495 & 0.031 \\
& 12 & 48 & 6.640 & 1.4579 & 0.13536 & 0.041 \\
\hline \hline
\end{tabular}

Table 1: Simulation parameters [9].

with projection operators $\Lambda_{ \pm}=\left(1 \pm \gamma^{0}\right) / 2$. The charge conjugation symmetry leads to $\rho_{0}(\omega)=$ $\rho_{0}(-\omega), \rho_{\mathrm{s}}(\omega)=-\rho_{\mathrm{s}}(-\omega)$, and $\rho_{+}(\omega)=\rho_{-}(-\omega)=\rho_{0}(\omega)+\rho_{\mathrm{s}}(\omega)$ [4]. In the following analysis, we concentrate on a determination of $\rho_{ \pm}(\omega)$ instead of $\rho_{0, \mathrm{~s}}(\omega)$, because excitation properties of quarks are more apparent in these channels. The spectral functions $\rho_{ \pm}(\omega)$ are neither even nor odd functions. In the chiral limit, however, $\rho_{\mathrm{s}}$ vanishes and $\rho_{ \pm}(\omega)$ become even functions. In analogy to Eq. (2.3) we introduce the decomposition of the correlation function $S(\tau)$ as $S(\tau)=S_{+}(\tau) \Lambda_{+} \gamma^{0}+S_{-}(\tau) \Lambda_{-} \gamma^{0}$, where $S_{ \pm}$are related through $S_{+}(\tau)=S_{-}(\beta-\tau)$.

For free quarks with scalar mass $m$ the spectral functions, $\rho_{ \pm}(\omega)=\pi \delta(\omega \mp m)$, have quark and anti-quark poles at $\omega= \pm m$, respectively. In the high temperature limit, additional poles, corresponding to the plasmino, appear at negative energy for $\rho_{+}(\omega)$ and positive energy for $\rho_{-}(\omega)$ [4, 5]. In Fig. 1, we show $\rho_{+}(\omega)$ in the Yukawa model with a massless boson at finite $T$ for various values of $m / T$ [4, 6]. One sees that the shape of $\rho_{+}(\omega)$ changes continuously between these two limits as $m / T$ varies.

To extract the spectral function $\rho_{+}(\omega)$ from $S(\tau)$ using Eq. (2.2), we assume that $\rho_{+}(\omega)$ can be described by a two-pole ansatz,

$$
\rho_{+}(\omega)=Z_{1} \delta\left(\omega-E_{1}\right)+Z_{2} \delta\left(\omega+E_{2}\right)
$$

where the residues $Z_{1,2}$ and energies $E_{1,2}>0$ have to be determined from a fit to $S_{+}(\tau)$. The poles at $\omega=E_{1},-E_{2}$ correspond to the normal and plasmino modes, respectively [4]. 


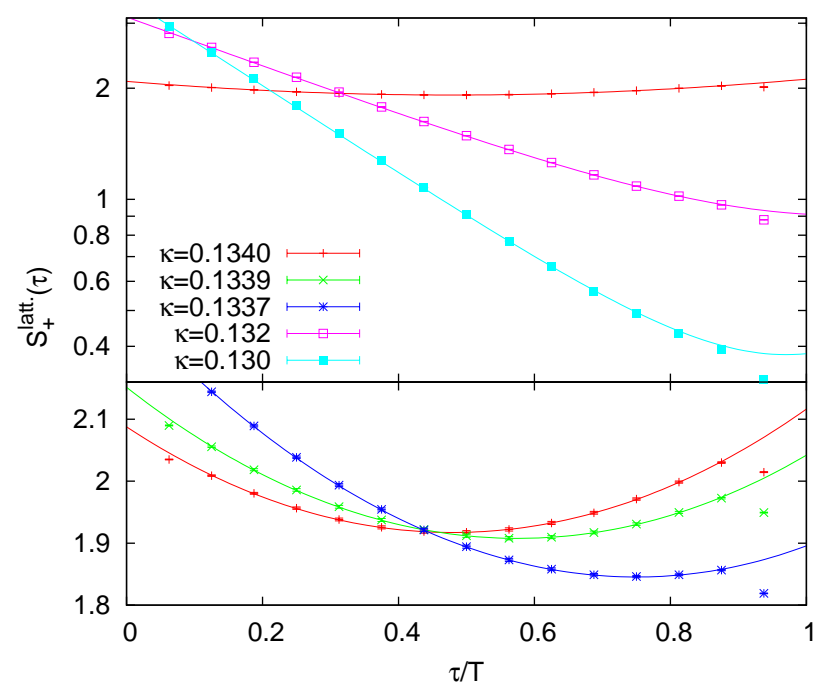

Figure 2: The lattice correlation function $S_{+}^{\text {latt. }}(\tau)$ at $T=3 T_{c}$ for the lattice of size $64^{3} \times 16$ with various values of $\kappa$, and the fitting result with the ansatz Eq. (2.4).

The correlation function $S(\tau)$ has been calculated at two values of the temperature, $T=1.5 T_{c}$ and $3 T_{c}$, in quenched QCD using non-perturbatively improved clover Wilson fermions [7, 8]. To control the dependence of our results on the finite lattice volume, $N_{\sigma}^{3} \times N_{\tau}$, and lattice spacing, $a$, we analyze the quark propagator on lattices of three different sizes. The gauge field ensembles used for this analysis have been generated and used previously by the Bielefeld group to study screening masses and spectral functions [9]. The different simulation parameters are summarized in Table 1 [9]. For each lattice size, 51 configurations have been analyzed. Quark propagators have been calculated after fixing each gauge field configuration to Landau gauge. For this we used a conventional minimization algorithm with a stopping criterion, $(1 / 3) \operatorname{tr}\left|\partial_{\mu} A^{\mu}\right|^{2}<10^{-11}$. In the Wilson fermion formulation the bare mass, $m_{0}$, is related to the hopping parameter $\kappa$, through the standard relation $m_{0}=\left(1 / \kappa-1 / \kappa_{c}\right) /(2 a)$, where $\kappa_{c}$ denotes the critical hopping parameter corresponding to the chiral limit, or vanishing quark mass.

\section{Numerical Results}

In Fig. 2, we show the numerical results for $S_{+}^{\text {latt. }}(\tau)$ for several values of $\kappa$ calculated on a lattice of size $64^{3} \times 16$ at $T=3 T_{c}$. One sees that the shape of $S_{+}^{\text {latt. }}(\tau)$ approaches that of a single exponential function for smaller $\kappa$, while it becomes symmetric as $\kappa$ approaches $\kappa_{c}$. In the vicinity of the wall source, i.e. at small and large $\tau$, we see deviations from this generic picture which can be attributed to distortion effects arising from the presence of the source. We thus exclude points with $\tau<\tau_{\min }$ and $N_{\tau}-\tau<\tau_{\min }$ from our fits to the ansatz given in Eq. (2.4). The resulting correlation functions obtained from correlated fits with $\tau_{\min }=3$ are shown in Fig. 2. One sees

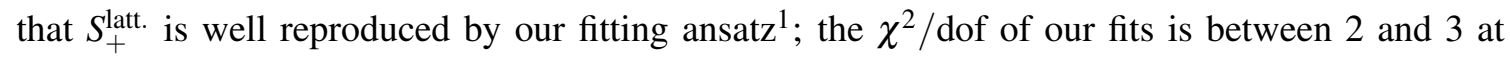

\footnotetext{
${ }^{1}$ We also checked that fits based only on a single pole ansatz lead to unacceptable large $\chi^{2} /$ dof.
} 


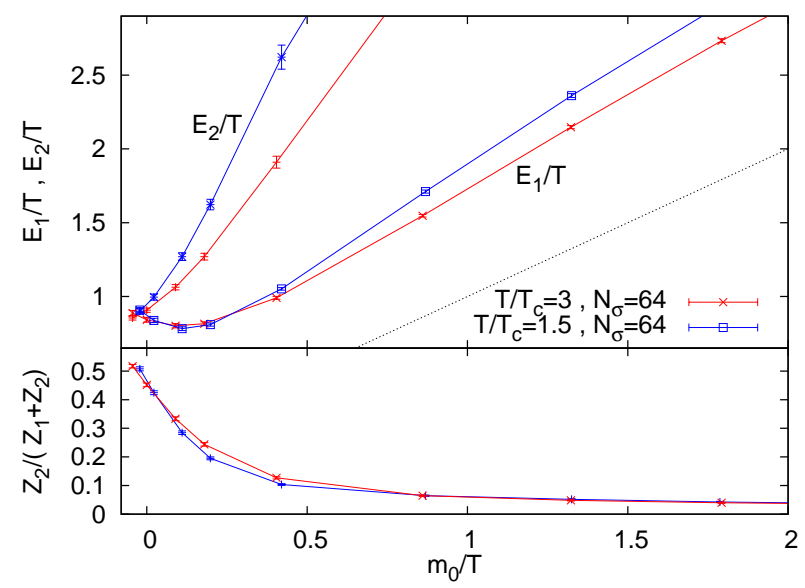

Figure 3: The bare quark mass dependence of fitting parameters $E_{1,2}$ and $Z_{2} /\left(Z_{1}+Z_{2}\right)$ at $T=1.5 T_{c}$ and $3 T_{c}$ for lattice $64^{3} \times 16$.

$1.335 \lesssim \kappa \lesssim 1.34$, while it gradually increases as $\kappa$ becomes smaller than $\kappa=1.335$. A similar behavior is also observed for our other lattice sizes [6].

In Fig. 3, we show the dependence of $E_{1,2}$ and $Z_{2} /\left(Z_{1}+Z_{2}\right)$ on the bare quark mass $m_{0}$ for $T=1.5 T_{c}$ and $3 T_{c}$. The results have been obtained from two-pole fits on lattices of size $64^{3} \times 16$. Errorbars have been estimated from a Jackknife analysis. The dotted line in this figure denotes the pole mass determined from the bare lattice mass. The figure shows that the ratio $Z_{2} /\left(Z_{1}+Z_{2}\right)$ becomes larger with decreasing $m_{0}$ and eventually reaches 0.5 . The hopping parameters satisfying $Z_{1}=Z_{2}$ are $\kappa_{c}^{\prime}=0.133974(10)$ for $T=3 T_{c}$ and $\kappa_{c}^{\prime}=0.134991(9)$ for $T=1.5 T_{c}$, which are consistent with the values for $\kappa_{c}$ given in Table 1]. The latter had been obtained in [9] from a fit to critical hopping parameters determined in [8] from the vanishing of the isovector axial current. The numerical results obtained on $64^{3} \times 16$ lattices show that $E_{1}$ and $E_{2}$ are equal within statistical errors at $\kappa=\kappa_{c}^{\prime}$. The spectral function $\rho_{+}(\omega)$ thus becomes an even function at this point; the quark propagator becomes chirally symmetric despite the presence of a thermal mass, $m_{T} \equiv E_{1}=E_{2}$. From Fig. 3, one also finds that the ratio $m_{T} / T$ is insensitive to $T$ in the temperature range analyzed in this work, while it is slightly larger for lower $T$.

As $m_{0}$ becomes larger, $Z_{2} /\left(Z_{1}+Z_{2}\right)$ decreases and $\rho_{+}(\omega)$ is eventually dominated by a singlepole. One sees that $E_{1}$ has a minimum at $m_{0}>0$, while $E_{2}$ is an increasing function of $m_{0}$. In the one-loop approximation, the peaks in $\rho_{+}(\omega)$ corresponding to $E_{1}\left(E_{2}\right)$ are monotonically increasing (decreasing) function of $m_{0} / T$ [4, 6]. The quark mass dependence of poles found here thus is qualitatively different from the perturbative result. We find, however, that slope of $E_{2}$ as function of $m_{0} / T$ decreases with increasing $T$. This may suggest that the perturbative behavior could eventually be recovered at much larger temperatures.

In order to check the dependence of our results on the lattice spacing and finite volume, we analyzed the quark propagator at $T=3 T_{c}$ for three different lattice sizes. Results for $E_{1}$ and $E_{2}$ are shown in Fig. 4. Comparing the results obtained on lattices with different lattice cut-off, $a$, but same physical volume, i.e. $64^{3} \times 16$ and $48^{3} \times 12$, one sees that any possible cut-off dependence is statistically not significant in our analysis. On the other hand we find a clear dependence of the 


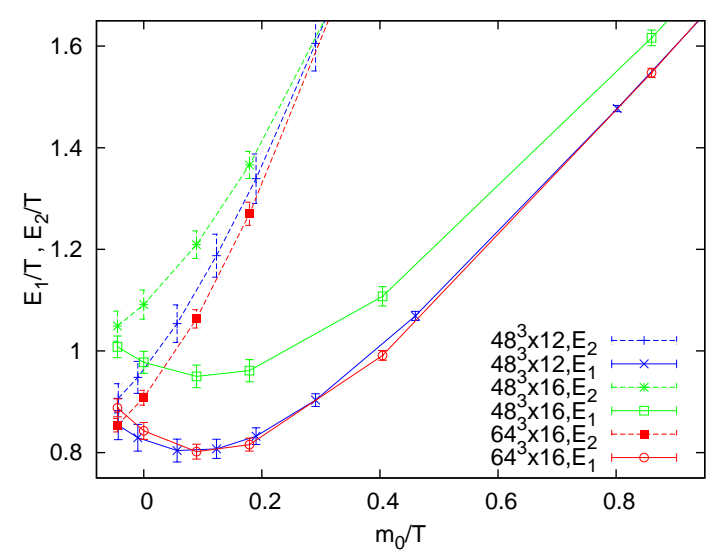

Figure 4: The bare quark mass dependence of parameters $E_{1}, E_{2}$ at $T=3 T_{c}$ for lattices of size $64^{3} \times 16$, $48^{3} \times 16$ and $48^{3} \times 12$.

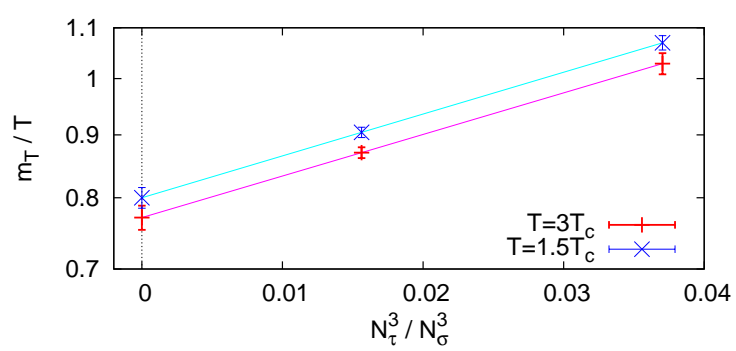

Figure 5: The bare quark mass dependence of fitting parameters $E_{1,2}$ and $Z_{2} /\left(Z_{1}+Z_{2}\right)$ at $T=1.5 T_{c}$ and $3 T_{c}$ for lattice $64^{3} \times 16$.

quark energy levels on the spatial volume; when comparing lattices with aspect ratio $N_{\sigma} / N_{\tau}=3$ and 4 we find that the energy levels, $E_{1,2}$, drop significantly. A similar behavior is observed also at $T=1.5 T_{c}$.

The presence of a strong volume dependence of the quark propagator is not unexpected. In fact, the thermal quark mass arises as collective effect of low momentum gluons; gluons at the soft scale $p \lesssim g T$ play a crucial role to give rise to the thermal mass at high temperatures [3]. However, on lattices with given aspect ratio $N_{\sigma} / N_{\tau}$ low momentum gluons are cut-off. The lowest non-vanishing gluon momentum is, $p_{\min } / T=2 \pi\left(N_{\tau} / N_{\sigma}\right)$, which still is larger than unity on lattices with aspect ratio $N_{\sigma} / N_{\tau}=4$. The situation may, nonetheless, be somewhat better in the temperature range explored here as the temperature dependent coupling $g(T)$ is larger than unity. An analysis of quark spectral functions on lattices with even larger spatial volume is needed in the future to properly control effects of small momenta. We attempted to estimate the thermal mass in the $V \rightarrow \infty$ limit by extrapolating the results obtained for two different volumina. Defining $m_{T} \equiv\left(Z_{1} E_{1}+Z_{2} E_{2}\right) /\left.\left(Z_{1}+Z_{2}\right)\right|_{\kappa=\kappa_{c}}$ and assuming the volume dependence of $m_{T}$ as $m_{T}\left(N_{\tau} / N_{\sigma}\right)=$ $m_{T}(0) \exp \left(N_{\tau}^{3} / N_{\sigma}^{3}\right)$, we obtain $m_{T}(0) / T=0.771(18)$ for $T=3 T_{c}$ and $m_{T}(0) / T=0.800(15)$ for $T=1.5 T_{c}$. This suggests that finite volume effects may still be of the order of $15 \%$ in our current 
analysis of $m_{T} / T$ (see Fig. 5). Despite these problems, our result clearly shows that light quarks near but above $T_{c}$ have a mass gap that is of collective nature similar to that in the perturbative regime.

\section{Summary}

In this study, we analyzed the quark spectral function at zero momentum for $T=1.5 T_{c}$ and $3 T_{c}$ as functions of bare quark mass $m_{0}$ in quenched lattice QCD with Landau gauge fixing. We found that the two-pole approximation for $\rho_{+}(\omega)$ well reproduces the behavior of the lattice correlation function. It is argued that the chiral symmetry of the quark propagator is restored at the critical value of $\kappa$ and the shape of the spectral function at this point takes a similar form as in the high temperature limit having normal and plasmino modes with thermal mass $m_{T}$. Meanwhile, $\rho_{+}(\omega)$ approaches a single-pole structure as $m_{0}$ is increased, as one can naturally deduce intuitively. The non-perturbative nature of thermal gauge fields is reflected in the behavior of poles as functions of $m_{0}$, which is qualitatively different from the perturbative result [4]. We also note that the ratio $m_{T} / T$ decreases slightly with increasing $T$, which is expected to happen at high temperature where $m_{T} / T$ should be proportional to a running coupling $g(T)$. Although results on the quark propagator are gauge dependent, we expect that our results for its poles suffer less from gauge dependence, because the success of the pole approximation for $\rho_{+}(\omega)$ indicates that the quark propagator has dynamical poles near the real axis, which are gauge independent quantities.

In the present study, we analyzed the quark spectral function in the quenched approximation. Although this approximation includes the leading contribution in the high temperature limit [3] and thus is valid at sufficiently high $T$, the validity of this approximation near $T_{c}$ is nontrivial. For example, screening of gluons due to the polarization of the vacuum with virtual quark antiquark pairs is neglected in this approximation. The coupling to possible mesonic excitations, which may cause interesting effects in the spectral properties of the quark [5], are not incorporated, either. The comparison of the quark propagator between quenched and full lattice simulations would tell us the strength of these effects near $T_{c}$.

\section{References}

[1] F. Karsch and M. Kitazawa, arXiv:0708.0299 [hep-lat].

[2] V.V. Klimov, Sov. J. Nucl. Phys. 33 (1981) 934 [Yad. Fiz. 33 (1981), 1734]; H.A. Weldon, Phys. Rev. D 28 (1983) 2007.

[3] M. Le Bellac, Thermal Field Theory (Cambridge University Press, Cambridge, England 1996).

[4] G. Baym et al.,Phys. Rev. D 46 (1992) 4043.

[5] M. Kitazawa, T. Kunihiro and Y. Nemoto, Phys. Lett. B 633 (2006) 269; Prog. Theor. Phys. 117 (2007) 103.

[6] F. Karsch and M. Kitazawa, in preparation.

[7] B. Sheikholeslami and R. Wohlert, Nucl. Phys. B 259 (1985) 572.

[8] M. Luscher et al., Nucl. Phys. B 491 (1997) 344.

[9] F. Karsch et al., Phys. Lett. B 530 (2002) 147; S. Datta et al., Phys. Rev. D 69 (2004) 094507. 